\title{
Clinical Guideline Highlights for the Hospitalist: Anaphylaxis Management in Adults and Children
}

\author{
Desirée C Burroughs-Ray, MD, MPH ${ }^{1,2 \star}$, Daniel R Wells, MD ${ }^{1,2}$, Mallie M Dennis, BS', Christopher D Jackson, MD
}

${ }^{1}$ Department of Internal Medicine, University of Tennessee Health Sciences Center, Memphis, Tennessee; ${ }^{2}$ Department of Pediatrics, University of Tennessee Health Sciences Center, Memphis, Tennessee.

GUIDELINE TITLE: Anaphylaxis-A 2020 practice parameter update, systematic review, and Grading of Recommendations, Assessment, Development and Evaluation (GRADE) analysis

RELEASE DATE: April 2020

PRIOR VERSION: Anaphylaxis - a 2019 practice parameter and GRADE analysis
DEVELOPER: American Academy of Allergy, Asthma \& Immunology (AAAAI) and the American College of Allergy, Asthma, and Immunology (ACAAI)

\section{FUNDING SOURCE: None}

TARGET POPULATION: Adult and pediatric patients with anaphylaxis naphylaxis, an acute, life-threatening allergic response, affects multiple organ systems and manifests variably. Anaphylaxis is likely taking place if one or more of the following occurs: (a) suddenonset skin and mucosal tissue swelling, (b) skin and mucosal abnormalities or respiratory or gastrointestinal symptoms after exposure to an allergen, or (c) reduced blood pressure after exposure to an allergen. With an estimated lifetime prevalence of up to $5.1 \%$, it is a significant cause of morbidity in adults and children. ${ }^{1}$ The 2020 anaphylaxis practice parameter update provides recommendations on treatment, prevention, and assessment of biphasic symptom risk in patients experiencing anaphylaxis. ${ }^{2}$ The guideline provides five key recommendations and four good-practice statements, which we have consolidated into five recommendations for this update.

\section{KEY RECOMMENDATIONS FOR THE HOSPITALIST}

Recommendation 1. All patients with suspected or confirmed anaphylaxis should be treated with epinephrine. (Good-practice statement)

Self-injectable epinephrine is the first-line treatment for anaphylaxis, with weight-based dosing of $0.15 \mathrm{mg} / \mathrm{kg}$ for children weighing less than $30 \mathrm{~kg}$ and $0.30 \mathrm{mg} / \mathrm{kg}$ for children weighing more than $30 \mathrm{~kg}$ and adults. Delayed administration of epinephrine can increase anaphylaxis-associated morbidity and mortality. After epinephrine administration, patients should be observed in a healthcare setting for symptom resolution.

*Corresponding Author: Desirée C. Burroughs-Ray, MD, MPH;

Email: dburroug@uthsc.edu; Telephone: 901-448-5814; Twitter: @DBR_thats_me.

Published online first January 20, 2021.

Received: June 1, 2020; Revised: October 12, 2020;

Accepted: October 22, 2020

๑ 2021 Society of Hospital Medicine DOI 10.12788/jhm.3553
Recommendation 2. For all patients, clinicians should assess the risk for developing biphasic anaphylaxis. (Conditional recommendation, very low quality of evidence)

Biphasic anaphylaxis is defined as the return of anaphylaxis symptoms after an asymptomatic period of at least 1 hour, all during a single instance of anaphylaxis. Biphasic anaphylaxis occurs in up to $20 \%$ of patients. ${ }^{3}$ Biphasic anaphylaxis is more likely among patients receiving repeated doses of epinephrine (odds ratio [OR], 4.82; 95\% Cl, 2.70-8.58), delayed epinephrine administration greater than 60 minutes $(\mathrm{OR}, 2.29 ; 95 \% \mathrm{Cl}$, 1.09-4.79), or a severe initial presentation $(\mathrm{OR}, 4.82 ; 95 \% \mathrm{Cl}$, 1.23-3.61). ${ }^{2}$ The presence of any of these risk factors raises the risk for developing biphasic anaphylaxis by $17 \% .{ }^{4}$ Severe anaphylaxis is characterized by life-threatening symptoms, including loss of consciousness, syncope or dizziness, hypotension, cardiovascular system collapse, or neurologic dysfunction from hypoperfusion or hypoxia after exposure to an allergen. ${ }^{5}$

Other risk factors for biphasic anaphylaxis in all ages include a widened pulse pressure, unknown anaphylaxis trigger, and cutaneous signs and symptoms. Drug triggers are also a risk factor in pediatric patients. ${ }^{2}$

Recommendation 3. All patients with anaphylaxis and risk factors for biphasic anaphylaxis should undergo extended clinical observation in a setting capable of managing anaphylaxis. (Conditional recommendation, very low quality of evidence)

All patients should be monitored for resolution of symptoms prior to discharge, regardless of age or severity at onset. Patients with all three of the following can be discharged 1 hour after symptom resolution because these three factors together have a $95 \%$ negative predictive value for biphasic anaphylaxis: nonsevere anaphylaxis, prompt response to epinephrine, and access to medical care. ${ }^{5}$ In contrast, extended observation of at least 6 hours should be offered to patients with increased risk of biphasic reactions. Patients who have potentially fatal 
underlying illnesses (eg, severe respiratory or cardiac disease), poor access to emergency medical services, poor selfmanagement skills, or inability to access epinephrine should also be considered for extended observation or hospitalization. Evidence is lacking to define the optimal observation time because extended biphasic reactions can occur from 1 to 78 hours after initial anaphylaxis symptoms. ${ }^{6}$

Given the lack of specific evidence around length of observation, there is an opportunity for shared decision-making. Every patient should receive education regarding trigger avoidance, reasons to seek care or activate emergency medical services, and warning signs of biphasic anaphylaxis. Additionally, selfinjectable epinephrine and an action plan detailing how and when to administer the epinephrine should be provided. Patients with anaphylaxis should follow up with an allergist.

Recommendation 4. Administration of glucocorticoids or antihistamines for prevention of biphasic anaphylaxis is not recommended. (Conditional recommendation, very low quality of evidence)

This guideline discourages glucocorticoids and antihistamines as a primary treatment as it may delay epinephrine administration. Despite treating the cutaneous manifestations of anaphylaxis, antihistamines fail to treat the life-threatening cardiovascular and respiratory symptoms. No clear evidence exists on whether antihistamines or glucocorticoids prevent biphasic anaphylaxis.

Recommendation 5. In adult patients receiving chemotherapy, premedication with antihistamine and/or glucocorticoid should be used to prevent anaphylaxis or infusion-related reactions for some chemotherapeutic agents in patients with no previous reaction to the drug. (Conditional recommendation, very low quality of evidence)

Premedication with antihistamines and/or glucocorticoids was associated with $51 \%$ reduced odds for anaphylaxis and infusion-related reactions to certain chemotherapy agents (pegaspargase, docetaxel, carboplatin, oxaliplatin, and rituximab) in adults who had not previously experienced a reaction to the drug $(\mathrm{OR}, 0.49 ; 95 \% \mathrm{Cl}, 0.37-0.66))^{2}$ However, this same benefit was not found with other chemotherapy agents for patients without a prior allergic reaction to the agent, which allows clinicians to defer premedication. The benefit of premedication with antihistamines and/or glucocorticoids to patients with prior anaphylactic reactions to chemotherapy agents was not evaluated in this guideline, nor was the role premedication plays in desensitization to chemotherapy.

\section{CRITIQUE}

This guideline was created by a panel of allergists, clinical immunologists, and methodologists using the GRADE (Grading of Recommendations, Assessment, Development and Evaluations) approach to draft recommendations. Conflicts of interest (COI) were disclosed by all panel members according to the American Academy of Allergy, Asthma, and Immunology (AAAAI) guidelines. The inclusion of many observational studies and meta-analyses improves the generalizability of the guideline. The authors highlighted the low certainty of evidence due to the lack of randomized controlled trials and significant heterogeneity of the included studies.

Some recommendations in the guideline have implications for costs of care. A recent economic analysis looked at cost-effectiveness for extended observation for anaphylaxis and found it was cost-effective only when patients were at increased risk for biphasic anaphylaxis. ${ }^{7}$ Although Recommendation 4 advises against the use of glucocorticoids for prevention of biphasic anaphylaxis, one retrospective cohort study demonstrated that glucocorticoid use was associated with decreased length of stay in children admitted with anaphylaxis. ${ }^{8}$ Therefore, the recommendation to avoid glucocorticoids for prevention of biphasic anaphylaxis could possibly increase hospital length of stay for children. The usefulness of dexamethasone to prevent biphasic anaphylaxis in children 3 to 14 months old is being evaluated in a randomized trial (ClinicalTrials.gov, NCT03523221).

\section{AREAS OF FUTURE STUDY}

Future research should better characterize risk factors for biphasic reactions to aid in clinical triage and diagnosis. Additional studies are needed to determine the optimal observation duration for patients experiencing anaphylactic reactions or requiring multiple doses of epinephrine. The role of premedication in patients receiving chemotherapy is poorly described, with few studies evaluating the benefit of premedication in patients with previous anaphylactic reactions.

Disclosures: The authors have no relevant financial conflicts of interest to disclose.

\section{References}

1. Wood RA, Camargo CA Jr, Lieberman P, et al. Anaphylaxis in America: the prevalence and characteristics of anaphylaxis in the United States. J Allergy Clin Immunol. 2014;133(2):461-467. https://doi.org/10.1016/j.jaci.2013.08.016

2. Shaker MS, Wallace DV, Golden DBK, et al. Anaphylaxis-a 2020 practice parameter update, systematic review, and Grading of Recommendations, Assessment, Development and Evaluation (GRADE) analysis. J Allergy Clin Immunol. 2020;145(4):1082-1123. https://doi.org/10.1016/j.jaci.2020.01.017

3. Lieberman $\mathrm{P}$, Camargo CA Jr, Bohlke K, et al. Epidemiology of anaphylaxis: findings of the American College of Allergy, Asthma and Immunology Epidemiology of Anaphylaxis Working Group. Ann Allergy Asthma Immunol. 2006;97(5):596-602. https://doi.org/10.1016/s1081-1206(10)61086-1

4. Kim TH, Yoon SH, Hong H, Kang HR, Cho SH, Lee SY. Duration of observation for detecting a biphasic reaction in anaphylaxis: a meta-analysis. Int Arch Allergy Immunol. 2019;179(1):31-36. https://doi.org/10.1159/000496092

5. Brown AF, Mckinnon D, Chu K. Emergency department anaphylaxis: a review of 142 patients in a single year. J Allergy Clin Immunol. 2001;108(5):861-866. https://doi.org/10.1067/mai.2001.119028

6. Pourmand A, Robinson C, Syed W, Mazer-Amirshahi M. Biphasic anaphylaxis: a review of the literature and implications for emergency management. Am J Emerg Med. 2018;36(8):1480-1485. https://doi.org/10.1016/j.ajem.2018.05.009

7. Shaker M, Wallace D, Golden DBK, Oppenheimer J, Greenhawt M. Simulation of health and economic benefits of extended observation of resolved anaphylaxis. JAMA Netw Open. 2019;2(10):e1913951. https://doi. org/10.1001/jamanetworkopen.2019.13951

8. Michelson KA, Monuteaux MC, Neuman MI. Glucocorticoids and hospital length of stay for children with anaphylaxis: a retrospective study. J Pediatr. 2015;167(3):719-724.e3. https://doi.org/10.1016/j.jpeds.2015.05.033 